\title{
Left-right symmetric composite Higgs model
}

\author{
Cong-Sen Guan, ${ }^{1,2}$ Teng Ma ${ }^{3},{ }^{3}$ and Jing Shu ${ }^{1,2,4,5,6}$ \\ ${ }^{1}$ CAS Key Laboratory of Theoretical Physics, Institute of Theoretical Physics, \\ Chinese Academy of Sciences, Beijing 100190, China \\ ${ }^{2}$ School of Physical Sciences, University of Chinese Academy of Sciences, \\ Beijing 100190, People's Republic of China \\ ${ }^{3}$ Physics Department, Technion-Israel Institute of Technology, Haifa, 3200003, Israel \\ ${ }^{4}$ CAS Center for Excellence in Particle Physics, Beijing 100049, China \\ ${ }^{5}$ Center for High Energy Physics, Peking University, Beijing 100871, China \\ ${ }^{6}$ School of Fundamental Physics and Mathematical Sciences, Hangzhou Institute for Advanced Study, \\ University of Chinese Academy of Sciences, Hangzhou 310024, China
}

(Received 4 December 2019; accepted 4 February 2020; published 24 February 2020)

\begin{abstract}
We find the exchange symmetry between left and right handed top quark in composite Higgs model with partial compositeness is efficient to soften the Higgs potential and reduce fine tuning. This symmetry can keep the Higgs potential in top sector invariant under trigonometric parity $\sin (h / f) \leftrightarrow \cos (h / f)$. Thus the Higgs quadratic divergences can be canceled entirely, resulting in a UV insensitive Higgs potential. We explicitly construct the minimal left-right symmetric model based on coset space $S O(6) / S O(5)$, which is locally isomorphic to $S U(4) / S p(4)$ and thus has well defined fermionic UV completion. This UV completion can automatically keep Higgs potential in the gauge sector finite even the gauge sector breaks this discrete symmetry. We find that the vector mesons can be very heavy, while the colored top partners are relatively light $(>1.5 \mathrm{TeV})$ to obtain a light Higgs.
\end{abstract}

DOI: 10.1103/PhysRevD.101.035032

\section{INTRODUCTION}

With the discovery of Higgs boson at LHC $[1,2]$, the Higgs mechanism [3-6] in the standard model (SM) is proven to successfully describe electroweak symmetry breaking (EWSB) at lower energy scale. Even though significant progress is achieved in understanding EWSB, some critical issues are still mysterious for us. Among them, the essential mystery of EWSB is how to stabilize the electroweak breaking scale against quantum correction.

One can achieve successful EWSB by introducing some new dynamics at some high energy scale, such as the $\mathrm{TeV}$ scale, to screen the quantum corrections from higher energy scales. Among these new physics theories, the composite Higgs model (CHM) [7-9] in which Higgs is a pseudoNambu-Goldstone boson (pNGB) of some strong dynamics can solve it in a tremendously simple way. Since Higgs is a bound state of strong dynamics, the quantum correction to Higgs potential from high energy scale is automatically screened by the constituents of Higgs, which results in the

Published by the American Physical Society under the terms of the Creative Commons Attribution 4.0 International license. Further distribution of this work must maintain attribution to the author(s) and the published article's title, journal citation, and DOI. Funded by SCOAP ${ }^{3}$. cut off of Higgs potential being reduced to the confinement scale $\Lambda$. Since pNGB Higgs potential is from loop corrections, if we further implement other mechanisms $[10,11]$, Higgs sensitivity to the confinement scale can be softened, and Higgs can be light.

However, the successful EWSB still requires tuning some parameters in the existing CHMs [12-15]. In these models, the main tuning is from the separation between EWSB scale $v$ and global symmetry breaking scale $f$ (called little hierarchy), $v \ll f$, to escape the constraints from electroweak precision tests $[16,17]$. What is worse is that they always suffer from double tuning [18], which makes realistic Higgs potential very difficult to be achieved. Recently it is found that the trigonometric parity [19] (TP) not only is the key ingredient to realizing neutral naturalness but also efficiently reduces the tuning in CHMs. The Higgs trigonometric parity can be induced not only through the $Z_{2}$ exchange symmetry between the SM sector and hidden sector (twin Higgs models) [19-22] but also through the maximal symmetry $[11,23]$.

In this paper, we find that the exchange symmetry between left-handed (LH) and right-handed (RH) SM fermions, called left-right (LR) symmetry, can eliminate the ultraviolet (UV) divergences and double tuning in the Higgs potential if partial compositeness [24] is implemented. Different from the composite twin Higgs models, this model can realize TP in the Higgs potential without 
introducing any extra partners in the elementary sector, but just through the left and right handed exchange symmetry of the SM fermions. As is well known, the TP is very efficient in eliminating UV divergence in the Higgs potential. This LR symmetry here enforces a UV insensitive Higgs potential in the top sector. The Higgs mass is correlated to the top partners' masses, so 4 percent tuning is needed to pay in order to get the light Higgs and heavy enough top partners in this model [25]. We find that the minimal coset to realize this LR symmetry is $S O(6) / S O(5)$ [28], which is locally isomorphic to $S U(4) / S p(4)$, and the latter has very well-defined pure fermionic UV completions based on the confining hypercolor gauge theory.

The structure of this paper is as follows. In Sec. II we introduce the general method to realize LR symmetry from the perspective of low energy effective theory. In Sec. III we explicitly realize this LR exchange symmetry in composite Higgs models based on the minimal coset $S O(6) / S O(5)$. In Sec. IV we briefly discuss the $S U(4) / S p(4)$ isomorphic version and the UV completion of this model. In Sec. V we analyze the EWSB and show that about 4 percent tuning is needed to achieve successful EWSB. Finally, in Sec. VI we briefly talked about the phenomenologies and showed that our model coincides with the direct search in the experiment. The Appendix contains the generators, specific form factors in effective Lagrangian and some comments on LR symmetric bottom sector.

\section{LEFT-RIGHT SYMMETRY IN EFFECTIVE LAGRANGIAN}

In twin Higgs models (THM) [20-22,29-31], Higgs divergences are canceled by introducing a $Z_{2}$ exchange symmetry between top and twin top to induce Higgs TP $\sin (h / f) \leftrightarrow \cos (h / f)$, which is very efficient to cancel the quadratic divergences of the ordinary top. In this section, we show that this $Z_{2}$ parity can also be directly realized by an exchange symmetry between the left and right handed top without introducing the twin sector. We first give a brief review of the trigonometric parity (TP) discussed in [19]. In pNGB Higgs models, any symmetric coset space $\mathcal{G} / \mathcal{H}$ contains a TP for any Goldstone boson $\pi^{i}$. This parity is the combination of $\pi / 2$ rotation in $\pi^{i}$ direction with the Higgs parity $\left(\pi^{i} / f \rightarrow-\pi^{i} / f+\pi / 2\right)$, which leads to the transformation as

$$
\cos \frac{\pi^{i}}{f} \leftrightarrow \sin \frac{\pi^{i}}{f}
$$

So if the left-right handed top exchange symmetry can contain the Higgs TP, then the Higgs potential will be

TABLE I. $\quad Z_{2}$ symmetries to induce Higgs TP in different models.

\begin{tabular}{lccc}
\hline \hline & Maximal symmetry & Twin Higgs & LR model \\
\hline$Z_{2}$ & $t_{L, R} \leftrightarrow t_{L, R}$ & $t_{L, R} \leftrightarrow \tilde{t}_{L, R}$ & $t_{L} \leftrightarrow t_{R}$ \\
Higgs TP & \multicolumn{3}{c}{$\sin (h / f) \leftrightarrow \cos (h / f)$} \\
\hline \hline
\end{tabular}

softened by this $Z_{2}$ symmetry, similar to THM. In Table I, we list different $Z_{2}$ symmetries used to induce Higgs TP in the maximal symmetry [11], twin Higgs [19] and this left-right (LR) symmetric model. It is straightforward to explicitly clarify the left-right symmetry and the mechanism to soft Higgs potential in low energy effective theory, as we discussed as follows.

The minimal coset space to realize the left-right (LR) symmetry is $S O(6) / S O(5)$. In this model, the electroweak (EW) gauge symmetry $S U(2)_{L} \times U(1)_{Y}$ is embedded in the $S O(4)$, which acts on the first four indices of $S O(6)$. More details about the gauge symmetry are shown in Appendix A. In unitary gauge, only the physical Higgs boson $h$ is uneaten and the pNGB Higgs matrix $U$ is given by

$$
U=\left(\begin{array}{cccc}
\mathbb{1}_{3} & & & \\
& \cos \frac{h}{f} & & \sin \frac{h}{f} \\
& & 1 & \\
& -\sin \frac{h}{f} & & \cos \frac{h}{f}
\end{array}\right),
$$

where $f$ is the global symmetry broken scale. The Higgs TP operator of this model is [19]

$$
P_{1}^{h}=R_{\pi / 2}^{h} V=\left(\begin{array}{cccc}
\mathbb{1}_{3} & & & \\
& & & -1 \\
& & 1 & \\
& -1 & &
\end{array}\right)
$$

where $R_{\pi / 2}^{h}=U(h / f=\pi / 2)$ is the $S O(2)$ rotation in Higgs direction with angle $\pi / 2$ and $V=\operatorname{diag}(1,1,1,1,1,-1)$ is the Higgs parity operator. It is easy to check $U$ transforms under $P_{1}^{h}$ as $U \rightarrow P_{1}^{h} U V=U\left(s_{h} \leftrightarrow c_{h}\right)$ with $s_{h} \equiv \sin (h / f)$ and $c_{h} \equiv \cos (h / f)$. In order to implement top LR symmetry, the EW doublet $q_{L}$ and singlet $t_{R}$ should be embedded in the same representation of $S O(6)$, such as fundamental representation:

$$
\Psi_{q_{L}}=\frac{1}{\sqrt{2}}\left(\begin{array}{c}
b_{L} \\
-i b_{L} \\
t_{L} \\
i t_{L} \\
0 \\
0
\end{array}\right), \quad \Psi_{t_{R}}=\frac{1}{\sqrt{2}}\left(\begin{array}{c}
0 \\
0 \\
0 \\
0 \\
t_{R} \\
i t_{R}
\end{array}\right),
$$

where we choose the embeddings of the singlet $t_{R}$ has a similar form as $t_{L}$ (for a reason for constructing LR symmetry). It is apparent that the incomplete embeddings of the bottom quark in Eq. (4) will bring some $Z_{2}$ breaking effects into the fermion sector. However, we can prove that this breaking effect can be neglected. More discussions about this $Z_{2}$ breaking effects, as well as constructing the LR symmetry in the bottom sector, are shown in the 
Appendix. D. Under the above setup, the parity operator exchanging $t_{L}$ and $t_{R}$ should be

$$
P \equiv P_{0} P_{1}^{h}=\left(\begin{array}{lll}
\mathbb{1}_{2} & & \\
& & \mathbb{1}_{2} \\
& \mathbb{1}_{2} &
\end{array}\right),
$$

where $P_{0}$ is the operator that exchanges the 3 rd and 5 th indices of $S O(6)$, and it is easy to check that $P_{0}$ acts trivially on $U, P_{0} U P_{0}=U$. The general effective Lagrangian invariant under global $S O(6)$ has the form

$$
\begin{aligned}
\mathcal{L}_{\text {eff }}= & \bar{\Psi}_{q_{L}} \not p\left(\Pi_{0}^{q}(p)+\Pi_{1}^{q}(p) \Sigma \Sigma^{\dagger}\right) \Psi_{q_{L}} \\
& +\bar{\Psi}_{t_{R}} \not\left(\Pi_{0}^{t}(p)+\Pi_{1}^{t}(p) \Sigma \Sigma^{\dagger}\right) \Psi_{t_{R}} \\
& +M_{1}^{t}(p) \bar{\Psi}_{q_{L}} \Sigma \Sigma^{\dagger} \Psi_{t_{R}}+\text { H.c. },
\end{aligned}
$$

where $\Sigma \equiv U . \mathcal{V}=\left(0,0,0, s_{h}, 0, c_{h}\right)^{T}$ is the linearly realized pNGB matrix which transforms under a global $S O(6)$ element $\mathbf{g}$ as $\Sigma \rightarrow \mathbf{g} \Sigma$, and $\mathcal{V}=(0,0,0,0,0,1)$ is the VEV breaking $S O(6)$ to $S O(5)$. From the effective Lagrangian we can formally infer that to implement the LR exchange symmetry, the form factors in left and right handed top kinetic terms should be equal:

$$
\Pi_{0}^{t}(p)=\Pi_{0}^{q}(p), \quad \Pi_{1}^{t}(p)=\Pi_{1}^{q}(p),
$$

which also implies that the underlying strong dynamics should be LR symmetric. Actually under the condition (7), one can easily check that the effective Lagrangian is indeed invariant under the LR transformations defined as

$$
\begin{aligned}
\Psi_{q_{L}, t_{R}} & \rightarrow P \Psi_{t_{R}, q_{L}}=\Psi_{q_{L}, t_{R}}\left(t_{L} \leftrightarrow t_{R}\right), \\
\Sigma & \rightarrow P \Sigma=\Sigma\left(s_{h} \leftrightarrow c_{h}\right) .
\end{aligned}
$$

One can further find that if the form factors satisfy the relations in Eq. (7), the kinetic terms in the effective Lagrangian exhibits an enlarged global $S O(6) \times S U(6)$ symmetry, where the QCD $S U(3)$ are embedded in $S U(6): \mathbf{6}=\mathbf{3}+\mathbf{3}$. While the effective Yukawa coupling explicitly breaks it. We can write the effective Lagrangian in $S O(6) \times S U(6)$ form as

$$
\begin{aligned}
\mathcal{L}_{\text {eff }}^{t}= & \operatorname{Tr}\left[\bar{\Psi}_{L R} \not p\left(\Pi_{0}(p)+\Pi_{1}(p) \Sigma \Sigma^{\dagger}\right) \Psi_{L R}\right] \\
& +\operatorname{Tr}\left[\bar{\Psi}_{L R} M_{1}^{t}(p) \Sigma \Sigma^{\dagger} \Psi_{R L}\right]
\end{aligned}
$$

where $\Psi_{L R}=\left(\Psi_{q_{L}}, \Psi_{t_{R}}\right)$ and $\Psi_{R L}=\left(\Psi_{t_{R}}, \Psi_{q_{L}}\right)$ are in $(\mathbf{6}, \mathbf{6})$ representation of $\mathcal{G}^{\prime} \equiv S O(6) \times S U(6)$ respectively. It is easy to find that the Yukawa coupling breaks this enlarged global symmetry. We can see these interactions are invariant under the LR $Z_{2}$ transformations in Eq. (8) which can be rewritten as

$$
\begin{aligned}
\Psi_{L R} & \leftrightarrow P \Psi_{R L}=\Psi_{L R}\left(t_{R} \leftrightarrow t_{L}\right), \\
\Sigma & \rightarrow P \Sigma=\Sigma\left(s_{h} \leftrightarrow c_{h}\right) .
\end{aligned}
$$

We have realized an exchange symmetry between $t_{L}$ and $t_{R}$, and this LR symmetry can trigger Higgs TP. The LR symmetry can be explicitly seen from the expansion of effective Lagrangian in terms of SM quarks:

$$
\begin{aligned}
\mathcal{L}_{\text {eff }}= & \bar{b}_{L} \not p \Pi_{0}^{q} b_{L}+\bar{t}_{L} \not p\left(\Pi_{0}^{q}+\frac{\Pi_{1}^{q}}{2} s_{h}^{2}\right) t_{L} \\
& +\bar{t}_{R} \not\left(\Pi_{0}^{t}+\frac{\Pi_{1}^{t}}{2} c_{h}^{2}\right) t_{R}+\frac{M_{1}^{t}}{2} \bar{t}_{L} t_{R} s_{h} c_{h}+\text { H.c. }
\end{aligned}
$$

It is apparently invariant under $t_{L} \leftrightarrow t_{R}, s_{h} \leftrightarrow c_{h}$ when imposing (7). Since the divergences of Higgs potential only come from the Higgs-dependant effective kinetic terms of top quark, and there are only two pNGB fields $(\Sigma)$ inserted in these terms (top quarks should be embedded in the simple representation of global symmetry), the quadratic divergent Higgs potential should be proportional to $s_{h}^{2}$ or $c_{h}^{2}$ and thus can be canceled by the Higgs TP induced from LR symmetry. We can directly calculate the one loop Higgs potential from top to prove our conclusion. In Euclidean momentum space, it is

$$
\begin{aligned}
V_{f}= & -2 N_{c} \int \frac{d^{4} p_{E}}{(2 \pi)^{4}} \log \left[1+\frac{\Pi_{1}^{q}\left(p_{E}\right)}{2 \Pi_{0}^{q}\left(p_{E}\right)} s_{h}^{2}+\frac{\Pi_{1}^{t}\left(p_{E}\right)}{2 \Pi_{0}^{t}\left(p_{E}\right)} c_{h}^{2}\right. \\
& \left.+\frac{\Pi_{1}^{t}\left(p_{E}\right) \Pi_{1}^{q}\left(p_{E}\right)}{4 \Pi_{0}^{t}\left(p_{E}\right) \Pi_{0}^{q}\left(p_{E}\right)} s_{h}^{2} c_{h}^{2}+\frac{\left|M_{1}^{t}\left(p_{E}\right)\right|^{2}}{4 p_{E}^{2} \Pi_{0}^{t}\left(p_{E}\right) \Pi_{0}^{q}\left(p_{E}\right)} s_{h}^{2} c_{h}^{2}\right],
\end{aligned}
$$

where $N_{c}$ is QCD color number. If the top mass is produced through partial compositeness, at large Euclidean momenta region, $\Pi_{0}^{q, t} \propto p_{E}^{0}, \Pi_{1}^{q, t} \propto p_{E}^{-2}$ and $M_{1}^{t} \propto p_{E}^{-2}$ [15]. So the Higgs quadratic divergence in above Coleman-Weinberg potential according to power counting is

$V_{f} \sim-2 N_{c} \int \frac{d^{4} p_{E}}{(2 \pi)^{4}}\left[\frac{\Pi_{1}^{q}\left(p_{E}\right)}{2 \Pi_{0}^{q}\left(p_{E}\right)} s_{h}^{2}+\frac{\Pi_{1}^{t}\left(p_{E}\right)}{2 \Pi_{0}^{t}\left(p_{E}\right)} c_{h}^{2}\right]$,

which indicates the Higgs quadratic divergence is only from top effective kinetic terms. Under the LR $Z_{2}$ symmetry, this term does not depend on the Higgs field, so Higgs quadratic divergence is eliminated. Notice that the Higgs potential from top effective Yukawa coupling is always finite, which is the only term allowed by the maximal symmetry [11].

In THM, the Higgs TP is induced by the exchange symmetry between top and its twin partner and Higgs potential is stabilized by the twin sector. While in LR symmetric CHM, the Higgs TP is induced by exchange symmetry between the LH and RH top and Higgs potential is stabilized by composite top partners. So partial 
compositeness is necessary to realize the natural Higgs potential for this model.

\section{THE MINIMAL LR SYMMETRIC COMPOSITE HIGGS MODEL $S O(6) / S O(5)$}

According to the discussions in the previous section, the minimal coset space to realize LR symmetry in top sector is $S O(6) / S O(5)$. However, the gauge sector does not exist LR symmetry, so the Higgs potential from gauge bosons' loop is quadratic divergent without introducing new resonances. But since the coset space $S O(6) / S O(5)$ is locally isomorphic to the coset $S U(4) / S p(4)$ which has very well defined fermionic UV completion, so the underlying strong dynamics can generally keep the Higgs potential from gauge boson loops converge at high energy scale, like pions in QCD. To mimic this high energy behavior in the effective theory, some heavy vector resonances should be introduced and impose Weinberg sum rules [15] on these resonances' decay constants and spectrum.

Since the set up of the gauge sector is the same as in paper [19], we do not give any more discussions about it. To be self-contained more details are shown in the Appendix A (also can be found in [19]).

\section{A. Realization of LR symmetry}

In Sec. II we assumed certain properties (7) in the lowenergy effective Lagrangian and find LR symmetry can cancel Higgs quadratic divergence. In this section we will explain how to realize the LR symmetry in $S O(6) / S O(5)$ CHM with partial compositeness.

As discussed in Sec. II, the ingredient that the fermions' masses are generated through partial compositeness is necessary for natural Higgs potential, so the composite top partners should be introduced. Since we have embedded LH doublet and RH singlet in the representation 6 of $S O(6)$, the composite operator $\mathcal{O}$ that mix with top quark is also supposed to be the same representation in order to mix with top quarks. Since the composite sector is $S O(5)$ invariant, $\mathcal{O}$ should be decomposed to a five-plet and a singlet multiplets of $S O(5), \mathcal{O} \rightarrow \Psi_{5}+\Psi_{1}$. The global $S O(6)$ invariant Lagrangian for elementary and composite fermions based on CCWZ formalism [32] can be constructed as

$$
\begin{aligned}
\mathcal{L}_{f}= & \bar{\Psi}_{\mathbf{5}}\left(i \nabla-M_{5}\right) \Psi_{\mathbf{5}}+\bar{\Psi}_{\mathbf{1}}\left(i \nabla-M_{1}\right) \Psi_{\mathbf{1}} \\
& +f \bar{\Psi}_{q_{L}} P_{R}\left(\epsilon_{q 1} U \Psi_{\mathbf{1}}+\epsilon_{q 5} U \Psi_{\mathbf{5}}\right) \\
& +f \bar{\Psi}_{t_{R}} P_{L}\left(\epsilon_{t 1} U \Psi_{\mathbf{1}}+\epsilon_{t 5} U \Psi_{\mathbf{5}}\right)+\text { H.c. }
\end{aligned}
$$

where $\nabla_{\mu}=\partial_{\mu}-i E_{\mu}$ is the covariant derivative of composite sector and $E_{\mu}$ is CCWZ $e$-symbol (we neglect the $U(1)_{X}$ charge $X$ with $Y=T_{R}^{3}+X$ in this model). The explicit embedding of composite resonance multiplets is

$$
\Psi_{5}=\frac{1}{\sqrt{2}}\left(\begin{array}{c}
i B-i X_{5 / 3} \\
B+X_{5 / 3} \\
i T+i X_{2 / 3} \\
-T+X_{2 / 3} \\
i T_{-}^{\prime}-i T_{+}^{\prime} \\
0
\end{array}\right), \quad \Psi_{1}=\frac{1}{\sqrt{2}}\left(\begin{array}{c}
0 \\
0 \\
0 \\
0 \\
0 \\
T_{-}^{\prime}+T_{+}^{\prime}
\end{array}\right)
$$

where the two EW doublets with hypercharge $1 / 6$ and $7 / 6$, $(T, B)$ and $\left(X_{5 / 3}, X_{2 / 3}\right)$, fill the full representation of custodial symmetry $S O(4)$ and $T_{+,-}^{\prime}$ are EW singlet with positive and negative $S O(2)_{\eta}$ charge respectively.

Before discussing the LR symmetry, we first need to figure out how the pNGB fields $U$ transform under the LR parity $P=P_{0} P_{1}^{h}$ we defined in Sec. II. Recall that $U$ transforms under the TP as ( $U$ in unitary gauge) $P_{1}^{h} U V=$ $U\left(s_{h} \leftrightarrow c_{h}\right)$ and $P_{0}$ acts trivially on $U$, so it is easy to derive that $\left(P_{0}^{2}=1\right)$

$$
P U=P_{0} P_{1}^{h} U=U\left(s_{h} \leftrightarrow c_{h}\right) P_{0} V .
$$

Use this identity, we find if and only if the linear mixing couplings of LH and RH top to the composite multiplets are correspondingly equal,

$$
\epsilon_{q 1}=\epsilon_{t 1}, \quad \epsilon_{q 5}=\epsilon_{t 5},
$$

the Lagrangian is invariant under the LR exchange symmetry defined as

$$
\begin{aligned}
U & \rightarrow P U V P_{0}=U\left(s_{h} \leftrightarrow c_{h}\right) \\
\Psi_{q_{L}} & \leftrightarrow P \Psi_{t_{R}}, \quad P_{R} \Psi_{\mathbf{1 , 5}} \leftrightarrow P_{0} V P_{L} \Psi_{\mathbf{1 , 5}} .
\end{aligned}
$$

Use the explicit matrix form of these parity operators, one can easily check that $P_{0} V$ is an element of the unbroken subgroup $S O(5)$, which means when it acts on the composite fermion multiplets, the effect is just a redefinition of each component (i.e., $\Psi_{1,5} \rightarrow \Psi_{1,5}^{\prime}$ ). So the LR exchange symmetry is a good symmetry of interactions between fermion and NGBs in Eq. (14), and it will result in the Higgs potential from these fermions' loops is exactly TP invariant. After integrating out the composite fermions, we can get the LR symmetric effective Lagrangian (6) satisfying Eq. (7). The explicit expressions of the general form factors are shown in the Appendix B.

In Sec. II, we demonstrated that the LR symmetry could cancel the quadratic divergence in Higgs potential based on the effective Lagrangian and partial compositeness. Here we can also analyze the Higgs potential directly from the composite Lagrangian (14). Notice that since the SM fermions are embedded in the fundamental representation of global symmetry, one $U$ field is always associated with 
one Yukawa coupling $\lambda \in f\left\{\epsilon_{q 1}, \epsilon_{q 5}, \epsilon_{t 1}, \epsilon_{t 5}\right\}$. According to power counting, the quadratic divergent terms are proportional to $\lambda^{2}$, so the quadratic divergent terms of Higgs potential from the fermion sector at most contain two powers of $U$, proportional to $s_{h}^{2}$ or $c_{h}^{2}$. So Higgs TP is enough to eliminate them. However, the log divergent terms can at most contain four powers of $U$, proportional to the fourth power of trigonometric function, so usually they cannot be eliminated by TP. To further eliminate these terms, one can, as did in [11], suppose the composite sector has fully $S O(6)$ global symmetry in Yukawa couplings and the composite mass terms explicitly break it to $H \equiv S O(5)$, or equivalently embed this model in the $S O(6) / S O(5)$ two-site moose. The above setup requires the mixing couplings to satisfy

$$
\epsilon_{q 1}=\epsilon_{q 5}, \quad \epsilon_{t 1}=\epsilon_{t 5} .
$$

So $\Psi_{5}$ and $\Psi_{1}$ can be combined to fill complete representation 6 of global $S O(6)$. The Lagrangian with LR symmetry and fully global $S O(6)$ invariant elementarycomposite mixing terms (two-site moose deconstruction) has the form as follows

$$
\begin{aligned}
\mathcal{L}_{f}= & \bar{\Psi}_{\mathbf{6}} i \nabla \Psi_{\mathbf{6}}-\frac{M_{5}+M_{1}}{2} \bar{\Psi}_{\mathbf{6}} \Psi_{\mathbf{6}}-\frac{M_{5}-M_{1}}{2} \bar{\Psi}_{\mathbf{6}} V \Psi_{\mathbf{6}} \\
& +f \epsilon \bar{\Psi}_{q_{L}} P_{R} U \Psi_{\mathbf{6}}+f \epsilon \bar{\Psi}_{t_{R}} P_{L} U \Psi_{\mathbf{6}}+\text { H.c. }
\end{aligned}
$$

Under this setting, the Higgs shift symmetry is only broken by the top partner's masses, so the leading Higgs potential should be proportional to mass square $M^{2}$ with $M \in\left\{M_{5}, M_{1}\right\}$. On the other hand, TP invariant Higgs potential at least contains four powers of $U$, thus is proportional to $f^{4} \epsilon^{4}$. Combining above analysis, we find that if the fermion-Higgs interactions are LR symmetric and the elementary-composite mixing terms are fully $S O(6)$ invariant [i.e., the Yukawa couplings satisfy both Eq. (17) and Eq. (19)] or equivalently is deconstructed on two-site moose, Higgs potential must be proportional to $f^{4} \epsilon^{4} M^{2}$, thus finite according to power counting.

The above discussion is very general and does not depend on the particular coset space. Below we summarize the general principle to construct a TP invariant Higgs potential through LR exchange symmetry. Higgs TP $P_{1}^{h}$ always exists in any symmetric coset space $G / H$. To implement the LR symmetry and preserve Higgs TP, the LH and RH top should be embedded in the same representation of $G$ in such a way: the L-R exchange operator $P$ can be written as $P=P_{0} P_{1}^{h}$, which contains Higgs TP operator. The LR symmetry requires the Yukawa couplings of LH and RH top to the composite resonances should be equal (i.e., chirality independent), which will make the form factors of $\mathrm{LH}$ and $\mathrm{RH}$ top kinetic terms equal in the lower-energy effective lagrangian. So this LR symmetry can preserve Higgs TP and can efficiently forbid $s_{h}^{2}$ and $c_{h}^{2}$ terms (actually $\sin ^{2}(2 N+1) h / f$ and $\cos ^{2}(2 N+1) h / f$ terms are all be forbidden, where $N$ is integer). If the quadratic divergent terms in Higgs potential are only proportional to these terms, TP can eliminate it. While to further make Higgs potential finite, the elementarycomposite mixing terms should be fully $G$ invariant in the composite side or deconstruct this model in two-site moose.

\section{UV COMPLETION FOR $S U(4) / S p(4)$}

As is well known, $S O(6) / S O(5)$ and $S U(4) / S p(4)$ are locally isomorphic, and $S U(4) / S p(4)$ has well-defined UV completion based on a purely fermionic hypercolor theory. Since the Higgs sector is the same as the model in [19], we just review the main results in this sector and then focus on the UV completion for LR symmetric partial compositeness sector.

\section{A. Some reviews on UV completion for Goldstone sector}

The $S U(4) / S p(4)$ breaking pattern can be realized by introducing four Weyl fermions $\psi_{i}$ with $i=1,2,3,4$. The hypercolor gauge group can be chosen as $S p(2 N)$, and these preons are in the fundamental representation of hypercolor group $[33,34]$. The first two fermions $\left(\psi_{1}, \psi_{2}\right)$ are arranged into the $S U(2)_{L}$ doublet with $U(1)_{\eta} \cong S O(2)_{\eta}$ charge $\mathbf{1}$, and the last two fermions $\left(\psi_{3}, \psi_{4}\right)$ are $S U(2)_{R}$ doublet with hypercharge $\mp \mathbf{1 / 2}$ respectively and $U(1)_{\eta}$ charge $\mathbf{- 1}$. Since the preons are in pseudoreal representation, their scalar condensate $\left\langle\psi_{i} \psi_{j}\right\rangle \neq 0$ is antisymmetric with respect to $S U(4)$ flavor symmetry, and thus it can break global symmetry $S U(4)$ to $S p(4)$. This broken pattern produces five NGBs and their quantum number is the same as the ones in $S O(6) / S O(5)$ under the unbroken electroweak vacuum. We choose this vacuum in the form of

$$
V=\left(\begin{array}{cc}
i \sigma_{2} & 0 \\
0 & -i \sigma_{2}
\end{array}\right) \text {. }
$$

In this vacuum, the automorphism map can be constructed as

$$
T^{a} \rightarrow-V T^{a T} V^{T}, \quad T^{\hat{a}} \rightarrow-V T^{\hat{a} T} V^{T},
$$

where $T^{a}$ and $T^{\hat{a}}$ are unbroken and broken generators. With this map, the linearly realized Goldstone field $\Sigma^{\prime}$ can be constructed as

$$
\Sigma^{\prime}=U^{2} V
$$

where $U$ is the Goldstone matrix. It transforms linearly under global symmetry as $\Sigma^{\prime} \rightarrow g \Sigma^{\prime} g^{T}$ with $g \in S U(4)$. 


\section{B. LR symmetric top sector in $S U(4) / S p(4)$}

Since the $S U(4) / S p(4)$ is locally isomorphic with $S O(6) / S O(5)$ and has UV completion of pure fermions and gauge bosons, we can translate the LR symmetric top sector into this coset to find the realization of LR symmetry in the UV completion. According to the correspondence between these two equivalent coset spaces, the LH top doublet and RH top are embedded in two indices antisymmetric representation 6 of $S U(4)$ :

$\Psi_{q_{L}}=\frac{1}{\sqrt{2}}\left(\begin{array}{cc}\mathbf{0} & Q \\ -Q^{T} & \mathbf{0}\end{array}\right), \quad \Psi_{t_{R}}=\frac{1}{\sqrt{2}}\left(\begin{array}{cc}i t_{R} \sigma^{2} & 0 \\ 0 & \mathbf{0}\end{array}\right)$

where all of these are four by four antisymmetric matrices and $Q=\left(q_{L}, \mathbf{0}_{2 \times 1}\right)$. The LR exchange symmetry requires the global symmetry in top sector is enlarged to $S U(4) \times S U(6)$, as the $S O(6) / S O(5)$ case. Based on this enlarged global symmetry, we can write the effective Lagrangian to show the LR symmetry explicitly:

$$
\begin{aligned}
\mathcal{L}_{\text {eff }}= & \Pi_{0}(p) \operatorname{Tr}\left[\bar{\Psi}_{L R} \not \Psi_{L R}\right]+\Pi_{1}(p) \operatorname{Tr}\left[\bar{\Psi}_{L R} \Sigma^{\prime}\right] \not p \operatorname{Tr}\left[\Psi_{L R} \Sigma^{\prime \dagger}\right] \\
& +M_{1}^{t}(p) \operatorname{Tr}\left[\bar{\Psi}_{L R} \Sigma^{\prime}\right] \operatorname{Tr}\left[\Psi_{R L} \Sigma^{\prime \dagger}\right]
\end{aligned}
$$

where $\Psi_{L R}=\left(\Psi_{q_{L}}, \Psi_{t_{R}}\right)$ and $\Psi_{R L}=\left(\Psi_{t_{R}}, \Psi_{q_{L}}\right)$ are in the $(\mathbf{6 , 6})$ representations of $S U(4) \times S U(6)$, and again $\Pi_{0,1}$ and $M_{1}^{t}$ are form factors. The effective interactions are invariant under the LR exchange symmetry:

$$
\begin{aligned}
\Sigma^{\prime} & \rightarrow P_{1} \Sigma^{\prime} P_{1}=\Sigma^{\prime}\left(s_{h} \leftrightarrow c_{h}\right) \\
\Psi_{L R} & \rightarrow P_{1} \Psi_{R L} P_{1}=\Psi_{L R}\left(t_{L} \leftrightarrow t_{R}\right) \\
\Psi_{R L} & \rightarrow P_{1} \Psi_{L R} P_{1}=\Psi_{R L}\left(t_{L} \leftrightarrow t_{R}\right),
\end{aligned}
$$

where the LR exchange operator $P_{1}$ is

$$
P_{1}=\left(\begin{array}{llll}
1 & & & \\
& & 1 & \\
& 1 & & \\
& & & -1
\end{array}\right) \text {, }
$$

which is an element of $S U(4)$. We can explicitly express the effective Lagrangian in terms of SM quarks as

$$
\begin{aligned}
\mathcal{L}_{\mathrm{eff}}^{t}= & \bar{b}_{L} \not \Pi_{0}(p) b_{L}+\bar{t}_{L} \not p\left(\Pi_{0}(p)+2 \Pi_{1}(p) s_{h}^{2}\right) t_{L} \\
& +\bar{t}_{R} \not p\left(\Pi_{0}(p)+2 \Pi_{1}(p) c_{h}^{2}\right) t_{R} \\
& +2 M_{1}^{t}(p) \bar{t}_{L} t_{R} s_{h} c_{h}+\text { H.c. }
\end{aligned}
$$

It is easy to see that this Lagrangian is LR symmetric and is equivalent to the effective Lagrangian in the $S O(6) / S O(5)$ case in Eq. (11) up to a redefinition of the form factors.

\section{UV completion for partial compositeness with LR exchange symmetry}

In the previous two subsections, we have roughly argued the UV completion for the gauge-Goldstone sector and realization of LR exchange symmetry in low-energy effective Lagrangian for the top sector. In this section we will show the UV completion for effective top Yukawa. We suppose the effective Lagrangian arises from integrating out composite fermions which linearly mix with elementary top. To produce these bound states, some colored preons should be introduced. The colored preons can be either fermions or scalars. But we find scalar preons can not realize LR symmetry in the fermion sector. So in the following, we will explain pure fermionic UV completion for partial compositeness.

As in [19], to form the fermionic bound states with preons $\psi_{i}$, the colored Weyl fermions $\chi_{L, R}$ in two indices anti-symmetric representation of the $S p(2 N)$ hypercolor gauge group should be introduced. Their quantum numbers under hypercolor and SM gauge symmetry $S p(2 N) \times$ $S U(3)_{c} \times S U(2)_{L} \times U(1)_{Y}$ is summarized in Table II. According to the quantum number of the preons, the wave functions for top partners $\Psi_{\mathbf{5 , 1}}$ are

$$
\left\{\Psi_{5 L}, \Psi_{1 L}\right\} \cong \chi_{L}(\psi \psi), \quad\left\{\Psi_{5 R}, \Psi_{1 R}\right\} \cong \chi_{R}(\psi \psi),
$$

where the bracket () denotes the Lorentz index contract of the fermions. As discussed in the $S O(6) / S O(5)$ model, the LR symmetry in the low-energy effective top couplings requires the composite partners' sector should be LR exchange invariant, see Eq. (18). To maintain this $Z_{2}$ symmetry in low-energy effective theory, the UV completion should be invariant under colored preons' LR exchange symmetry

$$
\chi_{L} \leftrightarrow \chi_{R}
$$

The flavor symmetry in the colored preon sector is $S U(6) \times$ $U(1)$ with respect to hypercolor group with $S U(3)_{c} \subset$ $S U(6)$. At low energy scale the hypercolor gauge interaction confine, the colored preons may condense with each other $\left\langle\chi_{i} \chi_{j}\right\rangle \neq 0$ with $\chi=\left\{\chi_{L}, \chi_{R}^{c}\right\}$. The colored preons are in 2-index antisymmetric representation of hypercolor, so the condensates are in the 2-index symmetric representation of $S U(6)$ global group, and thus the global symmetry would be broken into $S O(6)$, resulting in $\mathbf{2 0}$ colored pNGB. If the symmetric condensates preserve QCD, the quantum number of these pNGBs under $S U(3)_{c}$ is

TABLE II. The quantum numbers of the colored preons.

\begin{tabular}{ccccc}
\hline \hline & $S p(2 N)$ & $S U(2)_{L}$ & $U(1)_{Y}$ & $S U(3)_{c}$ \\
\hline$\chi_{L}$ & $\boxminus$ & 1 & $\frac{2}{3}$ & $\square$ \\
$\chi_{R}^{c}$ & $\boxminus$ & 1 & $-\frac{2}{3}$ & $\square$ \\
\hline \hline
\end{tabular}




$$
20=8+6+\overline{6}
$$

Since these pNGBs are colored, they can be produced through QCD with a big cross section at LHC, resulting in around $1 \mathrm{TeV}$ lower bounds for their masses [35]. However, their masses can be from several sources, such as QCD interactions, the mass of colored preons and fermion Yukawas. Combining these effects, its mass can be heavy enough to escape experiment searches. More studies on this UV completion can be found in [19].

\section{HIGGS POTENTIAL AND FINE TUNING}

In this section we fully calculate the Higgs potential and discuss the EWSB. According to Eq. (12), the contribution of fermion loops to Higgs potential could be parametrized as follows (up to $\mathcal{O}\left(s_{h}^{4}\right)$ order),

$$
V_{f}=-\gamma_{f} s_{h}^{2}+\beta_{f} s_{h}^{4},
$$

while the contribution from gauge loops is [19]

$$
V_{g}=\frac{3 f^{2}\left(3 g^{2}+g^{2}-2 g_{1}^{2}\right) m_{\rho}^{2} \ln 2}{64 \pi^{2}} s_{h}^{2} \equiv \gamma_{g} s_{h}^{2},
$$

where $m_{\rho}$ is the mass of gauge resonance. The gauge contribution to $s_{h}^{4}$ can be neglected since it is at $\mathcal{O}\left(g^{4}\right)$ order. So the total Higgs potential has the form as

$$
V=-\gamma s_{h}^{2}+\beta s_{h}^{4},
$$

where $\gamma=\gamma_{f}-\gamma_{g}$ and $\beta=\beta_{f}$. The overall $\gamma$ has to be positive for a realistic model, thus the potential has a minimum at $s_{h}^{2}=\frac{\gamma}{2 \beta} \equiv \xi$. The exact $Z_{2}$ symmetry in the fermion sector leads to $\gamma_{f}=\beta_{f}$ for the leading expression, so the natural $\xi$ from the fermion sector is about $\xi \approx 0.5$. In order to get an experimentally allowed small $\xi(\xi \ll 1)$ to realize correct EWSB, the fermionic and gauge contribution to $\gamma$ should have a cancellation, which brings some fine tuning to this model. For the realistic VEV $\xi \ll 1$, the top mass can be extracted from eq. (11) as

$$
m_{t}=\left.\frac{\left|M_{1}^{t}(p)\right| s_{h}}{\sqrt{2 \Pi_{0}(p)\left(2 \Pi_{0}(p)+\Pi_{1}(p)\right)}}\right|_{p \rightarrow 0}=y_{t} v,
$$

where $\Pi_{0,1}^{q}=\Pi_{0,1}^{t} \equiv \Pi_{0,1}, y_{t}$ and $v$ are the SM top Yukawa coupling and Higgs VEV. From the explicit expressions of form factors in Eq. (C1), one can find $M_{1}^{t}(p) \simeq M_{f} \Pi_{1}(p)$ where $M_{f}$ is a typical top partner mass. So we use Eq. (35), the leading expression of $\gamma_{f}$ and $\beta_{f}$ (up to $\mathcal{O}\left(y_{t}^{2}\right)$ ) can be estimated from Eq. (12) by power counting as

$$
\gamma_{f}=\beta_{f} \simeq \frac{4 N_{c}}{(4 \pi)^{2}} y_{t}^{2} f^{2} M_{f}^{2}
$$

Then the Higgs mass can be derived from the potential (34) as

$$
m_{h}^{2}=\frac{8 \beta \xi(1-\xi)}{f^{2}} \simeq \frac{2 N_{c} \xi(1-\xi) y_{t}^{2}}{\pi^{2}} M_{f}^{2} .
$$

We see that the Higgs mass is sensitive to the top partner's mass in this model, so the top partner cannot be too heavy for a light Higgs. For example, if we fix $\xi=0.03, m_{h}=$ $125 \mathrm{GeV}$ and $m_{t}=150 \mathrm{GeV}$, the top partner's mass is about $M_{f} \simeq 1.54 \mathrm{TeV}$.

The tuning can be quantified as [18]

$$
\Delta=\operatorname{Max}\left(\Delta_{i}\right), \quad \Delta_{i}=\left|\frac{\partial \ln \xi}{\partial \ln x_{i}}\right|,
$$

where $x_{i}$ are the free parameters in the model. Since the $Z_{2}$ symmetry has fixed $\gamma_{f} \approx \beta_{f}$, the main tuning is from the cancellation between $\gamma_{f}$ and $\gamma_{g}$. Moreover, $M_{f}$ is almost fixed by Higgs mass, so the main tuning comes from the variation of free parameters $m_{\rho}$ and $U(1)_{\eta}$ gauge coupling $g_{1}$. The explicit expression for maximal tuning $\Delta$ is

$$
\begin{aligned}
\Delta=\Delta_{m_{\rho}} & =\frac{1}{\xi}-2 \text { for } m_{\rho}<\frac{4 \pi m_{h} \sqrt{1-2 \xi}}{\sqrt{3 \xi(1-\xi)\left(3 g^{2}+g^{\prime 2}\right) \ln 2}} \\
\Delta=\Delta_{g_{1}} & =\frac{1-2 \xi}{\xi}\left(\frac{3 \xi(1-\xi)\left(3 g^{2}+g^{\prime 2}\right) m_{\rho}^{2} \ln 2}{8 \pi^{2} m_{h}^{2}(1-2 \xi)}-1\right) \text { for } \\
m_{\rho} & >\frac{4 \pi m_{h} \sqrt{1-2 \xi}}{\sqrt{3 \xi(1-\xi)\left(3 g^{2}+g^{\prime 2}\right) \ln 2}} .
\end{aligned}
$$

For $\xi=0.03$, the tuning is about $\Delta \simeq 30$ if the gauge resonance mass $m_{\rho}$ less than $5 \mathrm{TeV}$. When $m_{\rho}>5 \mathrm{TeV}$, the tuning will grow linearly with $m_{\rho}^{2}$ because the cancellation between $U(1)_{\eta}$ and SM gauge sector becomes significant and will dominate the tuning.

In Fig. 1, we show the exact numerical result of tuning $\Delta$ as a function of $m_{\rho}$ and $M_{T}$ for Higgs mass $m_{h} \in$ $[120,130] \mathrm{GeV}$ with $\xi=0.03$, where $M_{T}$ is the lightest top partner's mass and the red line is the analytical result
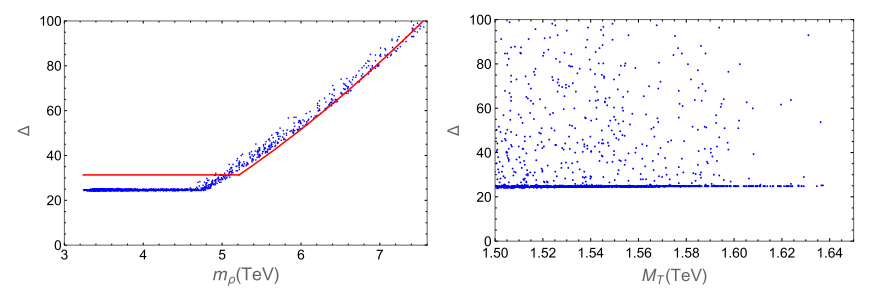

FIG. 1. Scatter plot for $\xi=0.03, m_{t} \in[140,170] \mathrm{GeV}$, $m_{h} \in[120,130] \mathrm{GeV}$ and $U(1)_{\eta}$ gauge boson mass $m_{B^{\prime}} \in\left[\frac{125}{2}, 1000\right] \mathrm{GeV}$. In the left (right) panel we show the tuning $\Delta$ as a function of $m_{\rho}$ (lightest top partner $M_{T}$ ). The red line corresponds to the estimation from Eq. (39). 


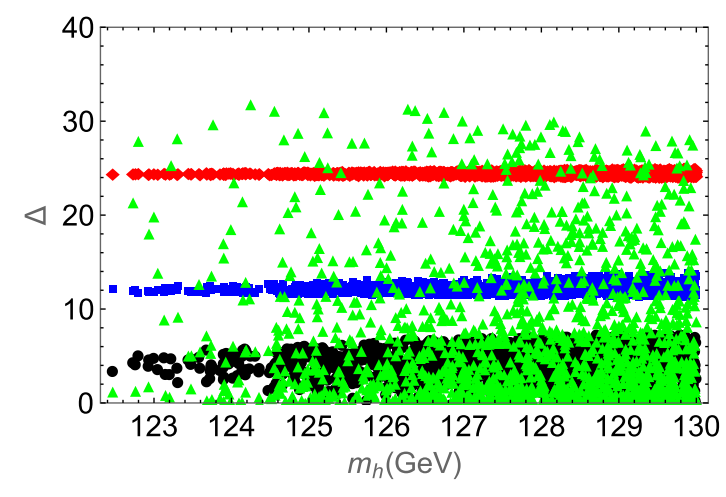

FIG. 2. Scatter plot of tuning $\Delta_{i}$ as a function of $m_{h}$ with $\xi$ fixed at 0.03 for the various free input parameters $x_{i}: M_{5}$ (black), $M_{1}$ (blue), $m_{\rho}$ (red) and $g_{1}$ (green). We choose the range of parameters as follows: $m_{t} \in[140,170] \mathrm{GeV}, m_{\rho} \in[2.5,5] \mathrm{TeV}, m_{B^{\prime}} \in$ $\left[\frac{125}{2}, 1000\right] \mathrm{GeV}$ and the lightest top partner's mass $M_{T}>1.5 \mathrm{TeV}$.

given by eq. (39). The deviation between Eq. (39) and the actual result comes from the higher order correction $\left(\mathcal{O}\left(y_{t}^{4}\right)\right)$ to $\beta_{f}$, which finally lead to $\beta_{f}>\gamma_{f}$. So the actual tuning of the model is 25 for a light $m_{\rho}$. The right panel shows that both low tuning $\Delta \sim 25$ and heavy top partners can be achieved for a light Higgs. In Fig. 2, we show the tuning for all the free parameters.

We should emphasize that these light composite resonances are consistent with the UV completion with a large hypercolor number $2 N$. Since the underlying hypercolor $S p(2 N)$ of our model can have very large color number, $2 \leq 2 N \leq 36$ for consistency [36], and the mass scale of composite resonances is $\sim 4 \pi f / \sqrt{2 N}$ in large $2 N$ limit [37], thus these resonances can be as light as $\mathcal{O}(f)$ (all the existing lattice calculations only focus on the small color region $2 N \leq 4$ [38-41] and thus is not applicable for our case). Moreover, in the large $2 N$ limit, if the underlying dynamics go to the conform window at a high energy scale, it can be holographically described by $5 \mathrm{D}$ weakly coupled theory through $A d S / C F T$ correspondence. Under this correspondence, the exact results of the 5D theory indicate that the masses of composite top partners and rho-mesons can be very light [14,42], at $\mathcal{O}(f)$. So the light resonances in our model can be generated in a large $2 N$ region.

\section{EXPERIMENTAL SIGNALS RELATED TO $U(1) \eta$ GAUGE BOSON}

We have never stopped looking for a SM singlet $U(1)$ gauge boson $Z^{\prime}$ in the experiment, and many BSM models have predicted the existence of such a $Z^{\prime}$. While in this paper $Z^{\prime}$ is just the $S O(2)_{\eta}$ gauge boson $B^{\prime}$. In this model $B^{\prime}$ could couple to two fermions through the covariant derivative terms. Since only the right handed top (bottom) and $T_{ \pm}^{\prime}$ carries the $U(1)_{\eta}$ charge, the primary decay channel of $B^{\prime}$ will be $t \bar{t}$ or $b \bar{b}$. In order to avoid the experimental restrictions from Higgs decay channel, we choose the $B^{\prime}$ mass in the range $[125 / 2,1000] \mathrm{GeV}$ when scanning the parameter space. We simulated the process $p p \rightarrow B^{\prime} \rightarrow$ $t \bar{t}(b \bar{b})$ and find that the cross section is below $0.31 \mathrm{pb}$ if $m_{B^{\prime}}<1 \mathrm{TeV}$, which is far below the experimental bounds $[43,44]$. So the $B^{\prime}$ mass we chosen in the model is reasonable and does not conflict to the direct search. Notice that the potential six top signals $p p \rightarrow t^{\prime} \bar{t}^{\prime} \rightarrow$ $B^{\prime} t B^{\prime} \bar{t} \rightarrow t t t \bar{t} \bar{t} \bar{t}$ ( $t^{\prime}$ generally refers to top partners) will also be the important channel to discover or constrain this model [45].

\section{CONCLUSIONS}

We introduced a $Z_{2}$ exchange symmetry between $\mathrm{LH}$ and RH fermions to the scenario of composite Higgs models, which trigger the $s_{h} \leftrightarrow c_{h}$ exchange symmetry in the Higgs potential, just like the twin Higgs models. This $Z_{2}$ is very efficient to reduce tuning because it could directly eliminate the quadratic divergences as well as the so-called "double tuning" in the Higgs potential. If we further impose some additional structure, such as two-site deconstruction or enlarge the global symmetry in the mixing terms between the elementary and composite sectors, the remaining logarithmic divergence could be eliminated and realize a finite Higgs potential with minimal tuning.

The concrete model we constructed is based on the symmetric coset $S O(6) / S O(5) \simeq S U(4) / S p(4)$, so there are 5 NGB fields which include a Higgs doublet and an SM singlet $\eta$. We gauge the SM groups as well as $U(1)_{\eta}$, so $\eta$ will be eaten by the $U(1)_{\eta}$ gauge boson $B^{\prime}$ after $U(1)_{\eta}$ is spontaneously broken by strong dynamics. One advantage of this model is that the isomorphic $S U(4) / S p(4)$ coset has very well defined pure fermionic UV completions. Although the gauge sector cannot realize such LR symmetry, the fermionic UV can force the composite resonances to satisfy Weinberg sum rules, and thus Higgs potential is converged. The successful EWSB $(\xi \ll 1)$ is realized by imposing the cancellation between the top and gauge sectors, which is the primary source of tuning in this model. The numerical calculation shows that a light Higgs can be realized with heavy top partners $(>1.5 \mathrm{TeV})$ and gauge resonances $(>3 \mathrm{TeV})$, which brings a $4 \%$ tuning for $\xi=0.03$.

\section{ACKNOWLEDGMENTS}

T. M. is supported in part by Project No. Y6Y2581B11 supported by the 2016 National Postdoctoral Program for Innovative Talents in China and also supported by the Israel Science Foundation (Grant No. 751/19), the United States-Israel Binational Science Foundation (BSF) (NSFBSF program Grant No. 2018683) and the Azrieli Foundation. J.S. is supported by the National Natural Science Foundation of China (NSFC) under Grants No. 11947302, No. 11690022, No. 11851302, No. 11675243, and 
No. 11761141011, and by the Strategic Priority Research Program of the Chinese Academy of Sciences under Grants No. XDB21010200 and No. XDB23000000.

\section{APPENDIX A: GAUGE SYMMETRY IN ELEMENTARY SECTOR}

The coset space $S O(6) / S O(5)$ contains 5 NGBs parametrized by $h_{i}$ and $\eta$ with $i=1,2,3,4$. The custodial symmetry $S O(4) \cong S U(2)_{L} \times S U(2)_{R}$ is embedded in the first four indices of $S O(6)$. Under the custodial symmetry, $h_{i}$ and $\eta$ is $S O(4)$ quartet and singlet, so $h_{i}$ corresponds to the SM Higgs doublet. These NGBs can be described by nonlinear sigma field

$$
U=\exp \left(i \sqrt{2} \pi^{\hat{a}} T^{\hat{a}} / f\right),
$$

where $\pi^{\hat{a}}=\left\{h_{i}, \eta\right\}$ and $T^{\hat{a}}$ are broken generators associated with the NGBs and normalized as $\operatorname{Tr}\left[T^{\hat{a}} T^{\hat{b}}\right]=\delta^{\hat{a} \hat{b}}$. The $S U(2)_{L} \times U(1)_{Y}$ with $U(1)_{Y} \subset S U(2)_{R}$ is gauged to be EW symmetry. Besides, we also gauge a $S O(2)_{\eta}$ which is the rotation between the last two indices of $S O(6)$. Since $S O(2)_{\eta}$ is in the broken direction, the associated NGB $\eta$ will be eaten by the corresponding massive gauge boson. So after EW symmetry is broken by vacuum misalignment, only one pNGB remains to play the role of Higgs and is denoted by $h$.

The gauge interactions of the pNGBs can be written in terms of linear realized $\Sigma$ field and the leading order Lagrangian is given by

$$
\mathcal{L}=\frac{f^{2}}{2}\left(D_{\mu} \Sigma\right)^{T} D^{\mu} \Sigma
$$

where $D_{\mu}=\partial_{\mu}-i g W_{\mu}^{a} T_{L}^{a}-i g^{\prime} B_{\mu} T_{R}^{3}-i g_{1} B_{\mu}^{\prime} T_{\eta}$ and $T_{L}^{a}$, $T_{R}^{3}$ and $T_{\eta}$ is generators of $S U(2)_{L}, U(1)_{Y}$ and $S O(2)_{\eta}$ embedded in $S O(6)$. After the EW symmetry is broken, the gauge bosons' masses are

$$
m_{W}=\frac{g f}{2} s_{h}, \quad m_{Z}=\frac{m_{W}}{\cos \theta_{W}}, \quad m_{B^{\prime}}=\frac{g_{1} f}{\sqrt{2}} c_{h},
$$

where $\theta_{W}$ is the weak mixing angle.

\section{APPENDIX B: GENERATORS}

In this appendix we list the explicit expressions of $S O(6)$ and $S U(4)$ generators we used in our model.

\section{1. $S O(6) / S O(5)$}

The $S O(6)$ generators could be classified in five broken generators in $S O(6) / S O(5)$ coset, six unbroken generators of custodial symmetry $S O(4) \cong S U(2)_{L} \times S U(2)_{R}$ and four unbroken generators of $S O(5) / S O(4)$ coset

$$
\begin{aligned}
T_{i j}^{\hat{a}} & =-\frac{i}{\sqrt{2}}\left(\delta^{\hat{a} i} \delta^{6 j}-\delta^{\hat{a} j} \delta^{6 i}\right), \\
T_{L, R i j}^{a} & =-\frac{i}{2}\left[\epsilon^{a b c} \delta^{b i} \delta^{c j} \pm\left(\delta^{a i} \delta^{4 j}-\delta^{a j} \delta^{4 i}\right)\right], \\
T_{i j}^{\alpha} & =-\frac{i}{\sqrt{2}}\left(\delta^{\alpha i} \delta^{5 j}-\delta^{\alpha j} \delta^{5 i}\right),
\end{aligned}
$$

where $\hat{a}$ from 1 to $5, a$ from 1 to 3 and $\alpha$ from 1 to 4 . The generator of $S O(2)_{\eta}$ is $T^{\hat{5}} \equiv T^{\eta}$.

\section{2. $S U(4) / S p(4)$}

The global symmetry breaking VEV $V$ in Eq. (21) breaks the global $S U(4)$ to $S p(4)$. The unbroken and broken generators satisfy the identity as follows

$$
T^{\alpha}=-V T^{\alpha T} V^{T}, \quad T^{\hat{\alpha}}=V T^{\hat{\alpha} T} V^{T} .
$$

So the explicit generators could be chosen as follows. The generators of custodial symmetry $S U(2)_{L} \times S U(2)_{R}$ are

$$
T_{L}^{a}=\frac{1}{2}\left(\begin{array}{cc}
\sigma^{a} & 0 \\
0 & 0
\end{array}\right), \quad T_{R}^{a}=\frac{1}{2}\left(\begin{array}{cc}
0 & 0 \\
0 & -\sigma^{a T}
\end{array}\right) .
$$

The remaining four unbroken generators are

$$
\frac{1}{2 \sqrt{2}}\left(\begin{array}{cc}
0 & i \sigma^{a} \\
-i \sigma^{a} & 0
\end{array}\right), \quad \frac{1}{2 \sqrt{2}}\left(\begin{array}{cc}
0 & \mathbb{1}_{2} \\
\mathbb{1}_{2} & 0
\end{array}\right) .
$$

The five broken generators are

$$
\begin{aligned}
T^{\hat{a}} & =\frac{1}{2 \sqrt{2}}\left(\begin{array}{cc}
0 & \sigma^{a} \\
\sigma^{a} & 0
\end{array}\right), \quad T^{\hat{4}}=\frac{1}{2 \sqrt{2}}\left(\begin{array}{cc}
0 & i \mathbb{1}_{2} \\
-i \mathbb{1}_{2} & 0
\end{array}\right), \\
T^{\eta} & =\frac{1}{2 \sqrt{2}}\left(\begin{array}{cc}
\mathbb{1}_{2} & 0 \\
0 & -\mathbb{1}_{2}
\end{array}\right),
\end{aligned}
$$

where as in $S O(6) / S O(5)$ case, $T^{\eta}$ is the generator of $U(1)_{\eta}$.

\section{APPENDIX C: FORM FACTORS IN EFFECTIVE LAGRANGIAN}

We can get the form factors in effective Lagrangian Eq. (11) by integrating out the fermion resonances from the global $S O(6)$ invariant Lagrangian Eq. (14). The explicit expression is

$$
\begin{aligned}
& \Pi_{0}^{q}(p)=1+\frac{f^{2} \epsilon_{q 5}^{2}}{M_{5}^{2}-p^{2}}, \quad \Pi_{1}^{q}(p)=\frac{f^{2} \epsilon_{q 1}^{2}}{M_{1}^{2}-p^{2}}-\frac{f^{2} \epsilon_{q 5}^{2}}{M_{5}^{2}-p^{2}} \\
& \Pi_{0}^{t}(p)=1+\frac{f^{2} \epsilon_{t 5}^{2}}{M_{5}^{2}-p^{2}}, \quad \Pi_{1}^{t}(p)=\frac{f^{2} \epsilon_{t 1}^{2}}{M_{1}^{2}-p^{2}}-\frac{f^{2} \epsilon_{t 5}^{2}}{M_{5}^{2}-p^{2}} \\
& M_{1}^{t}(p)=f^{2}\left(\frac{M_{1} \epsilon_{q 1} \epsilon_{t 1}}{M_{1}^{2}-p^{2}}-\frac{M_{5} \epsilon_{q 5} \epsilon_{t 5}}{M_{5}^{2}-p^{2}}\right) .
\end{aligned}
$$




\section{APPENDIX D: SOME COMMENTS ON BOTTOM SECTOR}

In the scenario of ordinary CHMs, it's hard to put the right handed bottom (top) into the top (bottom) sector because $t_{R}$ and $b_{R}$ have different hypercharge. So actually, LR exchange symmetry in kinetic terms is broken if including both the bottom and top sector interactions [see Eq. (4)]. However as discussed in [19], if the bottom is assigned in a proper representation, the $Z_{2}$ symmetry can be broken collectively such that the leading Higgs contributions from broken sector are at order $\mathcal{O}\left(\epsilon_{t}^{2} \epsilon_{b}^{2}\right)$, where $\epsilon_{t, b}$ are characteristic Yukawa coupling in top and bottom sectors. According to power counting, it is easy to find Higgs potential is still log divergent. To satisfy the above requirements, in the bottom sector we find the LH bottom doublet and RH bottom singlet should be put in the same representation as the top sector

$$
\Psi_{q_{L}}^{\prime}=\frac{1}{\sqrt{2}}\left(\begin{array}{c}
t_{L} \\
-i t_{L} \\
b_{L} \\
i b_{L} \\
0 \\
0
\end{array}\right), \quad \Psi_{b_{R}}=\frac{1}{\sqrt{2}}\left(\begin{array}{c}
0 \\
0 \\
0 \\
0 \\
b_{R} \\
i b_{R}
\end{array}\right) .
$$

Since the bottom Yukawa couplings are much smaller than top Yukawas $\epsilon_{b} \ll \epsilon_{t}$, the main contribution to Higgs potential is always from $Z_{2}$ preserving top sector and the $Z_{2}$ broken contributions, at $\mathcal{O}\left(\epsilon_{t}^{2} \epsilon_{b}^{2}\right)$, can be neglected. So in this paper we only focused on the top sector.
[1] G. Aad et al. (ATLAS Collaboration), Phys. Lett. B 716, 1 (2012).

[2] S. Chatrchyan et al. (CMS Collaboration), Phys. Lett. B 716, 30 (2012).

[3] F. Englert and R. Brout, Phys. Rev. Lett. 13, 321 (1964).

[4] P. W. Higgs, Phys. Rev. Lett. 13, 508 (1964).

[5] P. W. Higgs, Phys. Rev. 145, 1156 (1966).

[6] G. S. Guralnik, C. R. Hagen, and T. W. B. Kibble, Phys. Rev. Lett. 13, 585 (1964).

[7] D. B. Kaplan and H. Georgi, Phys. Lett. 136B, 183 (1984).

[8] H. Georgi and D. B. Kaplan, Phys. Lett. 145B, 216 (1984).

[9] M. J. Dugan, H. Georgi, and D. B. Kaplan, Nucl. Phys. B254, 299 (1985).

[10] N. Arkani-Hamed, A. G. Cohen, and H. Georgi, Phys. Rev. Lett. 86, 4757 (2001).

[11] C. Csaki, T. Ma, and J. Shu, Phys. Rev. Lett. 119, 131803 (2017).

[12] S. De Curtis, M. Redi, and A. Tesi, J. High Energy Phys. 04 (2012) 042.

[13] G. Panico and A. Wulzer, J. High Energy Phys. 09 (2011) 135.

[14] K. Agashe, R. Contino, and A. Pomarol, Nucl. Phys. B719, 165 (2005).

[15] D. Marzocca, M. Serone, and J. Shu, J. High Energy Phys. 08 (2012) 013.

[16] M. E. Peskin and T. Takeuchi, Phys. Rev. Lett. 65, 964 (1990).

[17] R. Barbieri, A. Pomarol, R. Rattazzi, and A. Strumia, Nucl. Phys. B703, 127 (2004).

[18] G. Panico, M. Redi, A. Tesi, and A. Wulzer, J. High Energy Phys. 03 (2013) 051.

[19] C. Csaki, T. Ma, and J. Shu, Phys. Rev. Lett. 121, 231801 (2018).

[20] Z. Chacko, H. S. Goh, and R. Harnik, Phys. Rev. Lett. 96, 231802 (2006).
[21] Z. Chacko, H. S. Goh, and R. Harnik, J. High Energy Phys. 01 (2006) 108; G. Burdman, Z. Chacko, H. S. Goh, and R. Harnik, J. High Energy Phys. 02 (2007) 009.

[22] N. Craig, S. Knapen, and P. Longhi, Phys. Rev. Lett. 114, 061803 (2015).

[23] C. Csaki, T. Ma, J. Shu, and J. H. Yu, arXiv:1810.07704.

[24] D. B. Kaplan, Nucl. Phys. B365, 259 (1991).

[25] This minimal tunning can be reduced by raising the quartic term [26] or suppress the quadratic term [27] in the Higgs potential.

[26] C. Csaki, C. S. Guan, T. Ma, and J. Shu, arXiv:1904.03191.

[27] C. Csaki, C. S. Guan, T. Ma, and J. Shu, arXiv:1910.14085.

[28] Ref. [15] chooses the Weinberg sum rule condition for CHMs based on $S O(5) / S O(4)$ that the Higgs UV divergence from the left-handed and right-handed fermions cancels. However, there is no real symmetry behind this Weinberg sum rule condition.

[29] M. Geller and O. Telem, Phys. Rev. Lett. 114, 191801 (2015); C. Csaki, M. Geller, O. Telem, and A. Weiler, J. High Energy Phys. 09 (2016) 146.

[30] M. Low, A. Tesi, and L. T. Wang, Phys. Rev. D 91, 095012 (2015).

[31] R. Barbieri, D. Greco, R. Rattazzi, and A. Wulzer, J. High Energy Phys. 08 (2015) 161.

[32] G. Panico and A. Wulzer, Lect. Notes Phys. 913, 1 (2016).

[33] J. Galloway, J. A. Evans, M. A. Luty, and R. A. Tacchi, J. High Energy Phys. 10 (2010) 086.

[34] G. Cacciapaglia and F. Sannino, J. High Energy Phys. 04 (2014) 111.

[35] G. Cacciapaglia, H. Cai, A. Deandrea, T. Flacke, S. J. Lee, and A. Parolini, J. High Energy Phys. 11 (2015) 201.

[36] G. Ferretti and D. Karateev, J. High Energy Phys. 03 (2014) 077.

[37] E. Witten, Nucl. Phys. B160, 57 (1979).

[38] E. Bennett et al., arXiv:1912.06505. 
[39] J. W. Lee, E. Bennett, D. K. Hong, C. J. D. Lin, B. Lucini, M. Piai, and D. Vadacchino, Proc. Sci., LATTICE2018 (2018) 192 [arXiv:1811.00276].

[40] E. Bennett, D. K. Hong, J. W. Lee, C.-J. D. Lin, B. Lucini, M. Piai, and D. Vadacchino, EPJ Web Conf. 175, 08011 (2018).

[41] A. Hietanen, R. Lewis, C. Pica, and F. Sannino, J. High Energy Phys. 07 (2014) 116.
[42] R. Contino and A. Pomarol, J. High Energy Phys. 11 (2004) 058.

[43] The ATLAS Collaboration, Eur. Phys. J. C 78, 565 (2018).

[44] The ATLAS Collaboration, Phys. Rev. D 98, 032016 (2018).

[45] H. Han, L. Huang, T. Ma, J. Shu, T. M. P. Tait, and Y. Wu, J. High Energy Phys. 10 (2019) 008. 